\title{
MOTIVACIÓN Y AUTO-REGULACIÓN EN LOS ESTUDIANTES CON DATH. NUEVOS APORTES DE LA NEUROIMAGINERÍA
}

\author{
Lady Meléndez Rodríguez ${ }^{1}$
}

Recibido: 23/07/2008

Aceptado: 4/11/2008

\begin{abstract}
Resumen
El déficit atencional con hiperactividad (DATH) es una condición genética, aunque también puede presentarse como síntoma de otro tipo de deficiencias. Dicho déficit ha significado siempre una situación difícil de controlar por parte de educadores y familiares, pero, sobre todo, por los mismos estudiantes. En su mayoría, los estudiantes con DATH no muestran discapacidad intelectual, aunque sí una serie de dificultades para aprender. Actualmente, la investigación fundamentada en el desarrollo de la tecnología en neuroimaginería ha permitido una mayor comprensión de la situación. Este trabajo expone y da a conocer aspectos que el uso de tecnología de punta en neuroimaginería permite confirmar, así como algunos que es posible superar o desechar; todos relacionados con la motivación, la auto-regulación y el aprendizaje colaborativo en función de un mejor control y aceptación social, del alcance exitoso de los objetivos de enseñanza y de guiar al estudiante con DATH hacia nuevos aprendizajes, inclusive en la educación a distancia.
\end{abstract}

\section{Palabras clave \\ -Inclusión • DATH • Neuroimaginería • Motivación • Auto-regulación • Aprendizaje colaborativo • Éxito.}

\begin{abstract}
The attention deficit disorder with hyperactivity (ADD) is a genetic condition, although it can also appear like a symptom of another type of deficiency. This deficit has always meant a difficult situation to control on the part of educators and family, but mainly, by such students. The majority of students with ADD do not show intellectual disabilities, although they do show different difficulties to learn. At the moment, the investigation based on the technology development of neuroimagery has allowed a greater understanding of the situation. This work shows aspects that the use of the latest technology in neuroimagery allows to confirm, other new aspects which are shared, as well as some that are able to overcome or even reject; all of them related with motivation, selfregulation and collaborative learning based on a better control and social acceptance, of the
\end{abstract}

\footnotetext{
${ }^{1}$ Encargada del Programa de Educación Especial de la Universidad Estatal a Distancia de Costa Rica.1melendez@uned.ac.cr
} 
successful achievement of the learning objectives and the guidance of the ADD student towards new learnings, even on education at a distance.

\section{Keywords}

$\bullet$ Inclusion • ADD • Neuroimagery • Motivation • Self-regulation • Colaborative learning Success.

El déficit atencional con hiperactividad (DATH) es de origen genético, pero también puede aparecer de manera secundaria como componente de otras deficiencias. Aunque dicho déficit no se elimina, su impacto sobre el aprendizaje y la vida cotidiana puede regularse para que el estudiante experimente procesos educativos cada vez más exitosos mediante la utilización de mediaciones efectivas, lo cual también pueden aplicarse con igual logro en la educación a distancia.

El DATH se caracteriza por la presencia de impulsividad inapropiada, dificultades de atención y muchas veces por actividad excesiva. Lo que lleva a formas de comportamiento que generan intolerancia social y experiencias adversas frecuentes, que actúan de manera negativa y directa sobre la autoestima y la motivación del alumno que las presenta.

Los procesos de entrada al aprendizaje son los que se muestran más comúnmente alterados en el estudiante con DATH y estos se refieren a los procesos relacionados con la selección, la atención y la concentración sobre los estímulos que el estudiante debe aprender.

Como selección se define la oportunidad con que cuenta el alumno para dirigirse, sensoperceptualmente, al objeto de estudio al que debe atender, o sea, en el que se integran sus distintos canales de senso-percepción aislando, al menos de la primera perspectiva, al resto de estímulos del ambiente. Esto debe hacerlo durante el tiempo suficiente que le permita 
decodificar, codificar y quizá conceptualizar los elementos o el todo que el mediador desea que aprenda, a lo que se denomina concentración. Estos procesos suelen estar alterados en los estudiantes con déficit atencional con hiperactividad o sin ella.

Mediante neuroimágenes ${ }^{1}$, ha sido posible observar que la corteza prefrontal y ciertas regiones de los ganglios basales son más pequeñas en los niños diagnosticados con DATH (Blakemore y Frith, 2007). Al respecto, es necesario repasar que la corteza prefrontal es indispensable para la planificación y la toma de decisiones, el control de la atención y de conductas inapropiadas, además de ordenar a los ganglios basales el momento oportuno para inhibir movimientos. Así, se infiere que muchos de los comportamientos propios de las personas con ese diagnóstico podrían deberse al crecimiento insuficiente de las estructuras neurales señaladas.

También, en estos casos se requiere de una terapia conjunta: terapia cognitiva para mejorar el autocontrol y la atención, terapia familiar para afrontar el problema de mejor manera, además del uso de fármacos pertinentes. Esta parece ser la mejor prescripción que se puede dar para regular la situación, aunque no para eliminarla del todo; ya que el DATH no sólo no desaparece, sino que el tipo congénito es heredable, no obstante, sí es posible atenuar y auto-regular sus efectos adversos sobre el aprendizaje y las experiencias cotidianas.

Cuando se trata de niños, Blakemore y Frith (2007) explican lo siguiente:

Es lógico que sea necesaria la experiencia para crear hábitos de atención sostenida; por otro lado, los adultos pueden favorecer el control de la conducta dando órdenes verbales y recordando al niño lo importante que es hacer esto o lo otro, así como 
ofreciendo recompensas adecuadas. Los modelos de rol pueden muy bien tener cierto impacto en el ajuste del autocontrol. (p.158)

FIGURA 1

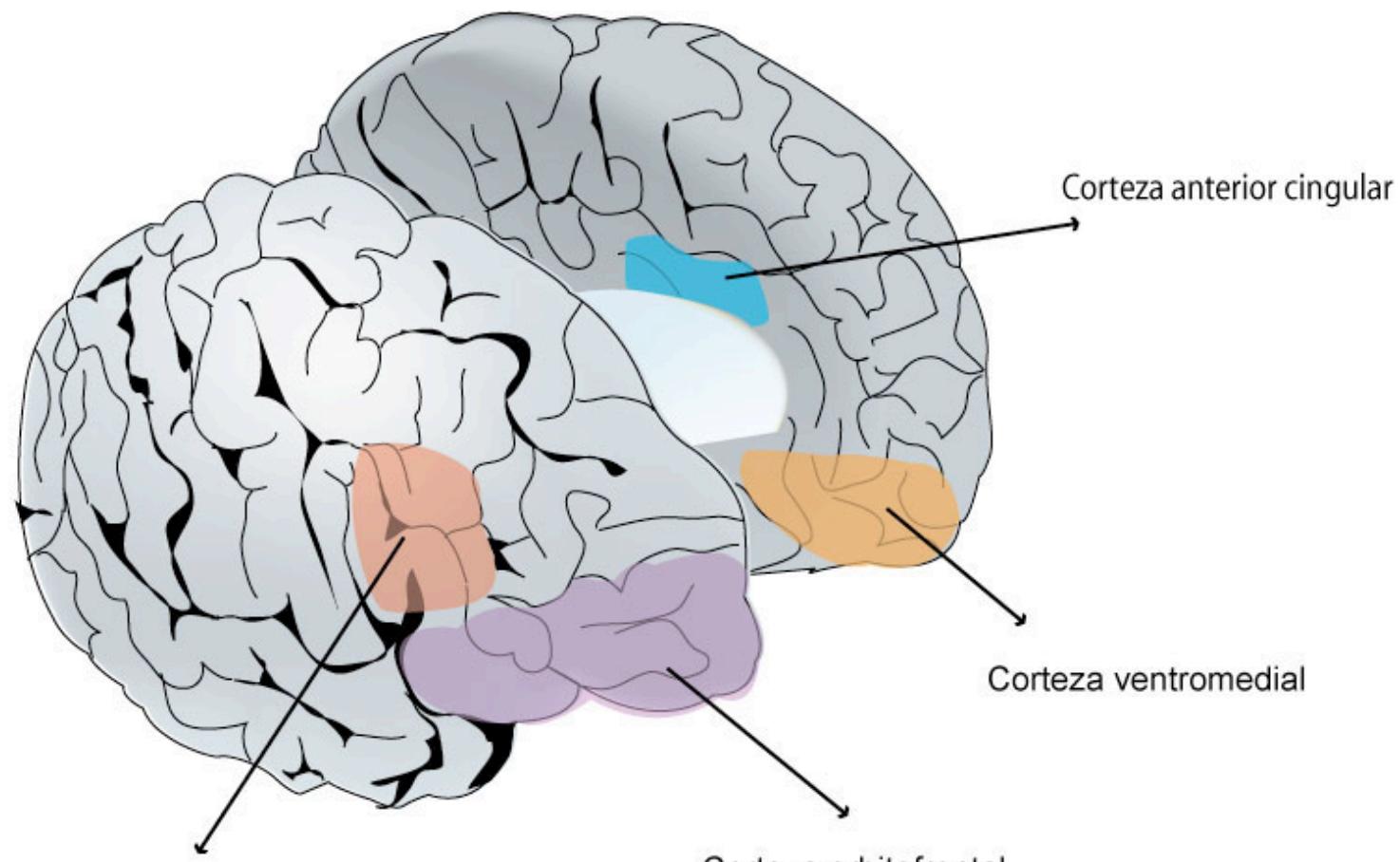

Corteza prefrontal dorsolateral

Corteza orbitofrontal

Figura tomada y adaptada de Carter (2002).

Un cerebro social adecuado depende, en gran medida, del funcionamiento del control de los impulsos que nos llevan a cometer acciones inapropiadas y a actuar en momentos inoportunos. También depende de prestar atención en el momento justo a las indicaciones propicias, inclusive a las que nos dictan nuestros pensamientos cuando están en sintonía con el comportamiento que los otros esperan y califican como adecuados. El buen funcionamiento integrado de las zonas marcadas en esta Figural es el encargado de lograrlo.

La persona con DATH comúnmente se enfrenta a ambientes negativos tanto en los contextos formales de aprendizaje, como durante el aprendizaje que se adquiere en la vida social. Lo que quizás constituye una de las razones que llevó a Carter (2002) a plantear su idea del cerebro social, para referirse a aquellas áreas cerebrales que frecuentemente se observan alteradas en una persona con DATH y que se destacan en la Figura 1. 


\section{Clasificación del DATH}

A diferencia de la clasificación elaborada por la American Psychiatric Association (DSMIV-TR, 1994), que define las dificultades mencionadas como trastornos clínicos para el aprendizaje y mentales del comportamiento, la Organización Mundial de la Salud, en su clasificación consultada mundialmente y publicada en el año 2001: Clasificación Internacional del Funcionamiento de la Discapacidad y de la Salud (CIF), define códigos y los relaciona con categorías específicas, bajo una tipología organizada en cuatro dimensiones que son: funciones corporales, estructuras corporales, actividades y participación y factores ambientales. La CIF reconoce que estas dimensiones interactúan entre sí y se determinan unas a otras, haciéndose más graves o más superables en cada caso.

Como ejemplo de una dificultad de aprendizaje típica, observada desde la clasificación mencionada, es posible exponer que si un estudiante tiene una alteración estructural que le impide poner atención sobre un estímulo en particular, pero aprende a realizarlo mediante estrategias de apoyo y uso de medicamentos pertinentes, el resto de las funciones y actividades que dependan de la atención, como instrumento de aprendizaje y de participación social, no tienen por qué verse alteradas. En la CIF, las funciones relacionadas con la atención se definen de la manera expresada en el Cuadro 1: 


\section{CUADRO 1}

\begin{tabular}{|l|}
\hline Código b140: Funciones de la atención* \\
\hline $\begin{array}{l}\text { Funciones mentales específicas que permiten centrarse en un estímulo externo o } \\
\text { experiencia interna durante el tiempo necesario. Incluyen: funciones relacionadas con el } \\
\text { mantenimiento de la atención, cambios del objeto de la atención, división de la atención, } \\
\text { compartir la atención; concentración y tendencia a estar distraído. }\end{array}$ \\
\hline
\end{tabular}

*El código b140 y la definición son dados por la OMS (2001).

\section{Alteración de las funciones ejecutivas}

Los aspectos más importantes que suelen verse afectados en forma negativa con la ocurrencia de los problemas mencionados son las funciones ejecutivas, relacionadas e igualadas por algunos autores con las funciones cognitivas superiores.

En este caso, más que como funciones preestablecidas, es necesario presentarlas como fenómenos mentales profundamente susceptibles al aprendizaje o, en su defecto, como funciones sensibles a la alteración de los procesos comprendidos en éste.

Blakemore y Frith (2007) ofrecen la siguiente lista de funciones ejecutivas: capacidad para inhibir conductas inadecuadas, planificar, seleccionar acciones, tener información en mente, hacer dos cosas a la vez, controlar emociones, tomar decisiones y resolver problemas. A esta lista Carter (2002) agrega la flexibilidad, mientras que Perea (2008) considera que las funciones ejecutivas son subyacentes a las funciones superiores en las que se encierran todos los procesos mentales más complejos: razonamiento, juicio crítico, atención-concentración, orientación, lenguaje, memoria, praxia y gnosia.

En nuestro caso, desde la lógica de la mediación pedagógica, hemos llamado funciones ejecutivas a dos ramas cognitivas fundamentales, a las habilidades de pensamiento y a las 
formas de razonamiento. A las primeras, las desglosamos como sigue: observación, predicción, planificación, resolución de problemas, toma de decisiones y comunicación asertiva (Meléndez, 2004); las cuales tienen en común con las listas anteriores que implican los más altos procesos cognitivos y se procesan, en su mayoría, en el lóbulo frontal del cerebro, frecuentemente afectado en las neuroimágenes de los estudiantes con DATH.

Es muy importante tomar en cuenta que los contenidos o la información que no ha sido aprendida en forma significativa son desechados muy fácilmente por el cerebro, no así las habilidades de pensamiento. Por tanto, la educación debería esforzarse más en desarrollar estas habilidades, pues luego le permitirán al estudiante recuperar y discernir mejor información desde cualquier fuente, y no gastar el tiempo en contenidos que, si no se utilizan, pronto se olvidan y requerirán cíclicamente de nuevos y costosos aprendizajes.

Por formas de razonamiento se entienden las distintas disposiciones mentales de ordenamiento categorial que utilizan los estudiantes cuando intentan resolver un problema. Se trata de disposiciones que pueden ser deductivas, inductivas, analógicas, silogísticas o complejas (Meléndez, 2004).

Es indispensable agregar que las formas de razonamiento inscriben representaciones mentales con las que se trazan esquemas que pueden ser transcritos gráficamente y utilizados para exponer, ilustrar y sintetizar información.

Entre los esquemas con capacidad de transcripción es posible identificar los gráficos de barras y fluxogramas, organigramas, mapas semánticos y conceptuales, cuadros sinópticos y líneas del tiempo, esquemas de llaves, matrices de doble entrada, uves heurísticas y otros 
que suelen aparecer en los materiales didácticos, aunque no necesariamente aplicados de manera congruente con la forma de razonamiento que se quiere desarrollar en el estudiante.

El reto para los mediadores didácticos es inducir cuáles son las formas de razonamiento involucradas en cada actividad, para no confundir a los estudiantes solicitándoles esquemas que organizan formas de razonamiento ajenas al ejercicio; como se observa, lamentablemente, en muchos libros de texto y en actividades de enseñanza. Por ejemplo, para comprender e interpretar un organigrama se requiere de razonamiento deductivo, para colocar piezas en un rompecabezas el razonamiento inductivo, para completar un mapa semántico de sinónimos o antónimos se utiliza el razonamiento analógico, para interpretar un esquema de llaves se debe echar mano del razonamiento silogístico y para construir un mapa conceptual es necesario acudir al razonamiento complejo (Meléndez, 2004).

La falta de atención, de control de conductas inapropiadas y de movimientos incesantes y muchas veces torpes son, en repetidas ocasiones, las causas de que los estudiantes con DATH no sólo vean afectados el resto de sus procesos de aprendizaje, sino de rechazo social y afectivo por parte de compañeros, maestros y hasta familiares. Lo que los lleva a caer en círculos interminables de dificultades como por ejemplo: fracaso-ansiedad, agresividad-rechazo, temor-timidez, timidez-omisión, bloqueo-fracaso.

\section{Motivación para aprender}

Es urgente descubrir estrategias adecuadas para lograr la motivación, sobre todo cuando el fracaso repetido y la ansiedad se han convertido en barreras infranqueables para que el estudiante pueda alcanzar aprendizajes exitosos. Estas estrategias son dignas de tomarse en 
cuenta en toda modalidad educativa, se haya o no identificado a algún estudiante con DATH en el ambiente de aprendizaje sobre el que se actúa didácticamente.

En la Figura 2 se ilustra una serie de componentes de tipo emocional que podrían interactuar en una situación de aprendizaje y lograr una actividad motivadora o no. Por ejemplo, al inicio de una tarea habrá siempre una forma de incertidumbre que tiene que ver con la primera impresión sobre la misma y es la percepción de la experiencia. Simplemente, el estudiante se coloca frente a la tarea y de acuerdo con sus aprendizajes previos, su estilo de aprendizaje, su personalidad y su memoria emocional, puede tener una apreciación positiva o negativa de la actividad. Enseguida se inicia una especie de lectura de un juego de expectativas, que involucran tanto la expectativa del estudiante sobre si él podrá cumplir con la tarea, como la expectativa que el alumno cree que el otro o los otros tienen con respecto a su rendimiento. A la vez, poseen un papel relevante las relaciones afectivas que el estudiante tenga con las personas cercanas a la actividad, que pueden ser sus compañeros, mediadores o familiares. Así, él o ella colocará mentalmente su autoestima en un grado que va a depender de qué tan apreciado o rechazado se sienta por las personas con las que interactúa. De la misma manera, entrarán a formar parte las condiciones emocionales actuales por las que el estudiante esté pasando, así como todo tipo de creencias personales y culturales. 
FIGURA 2

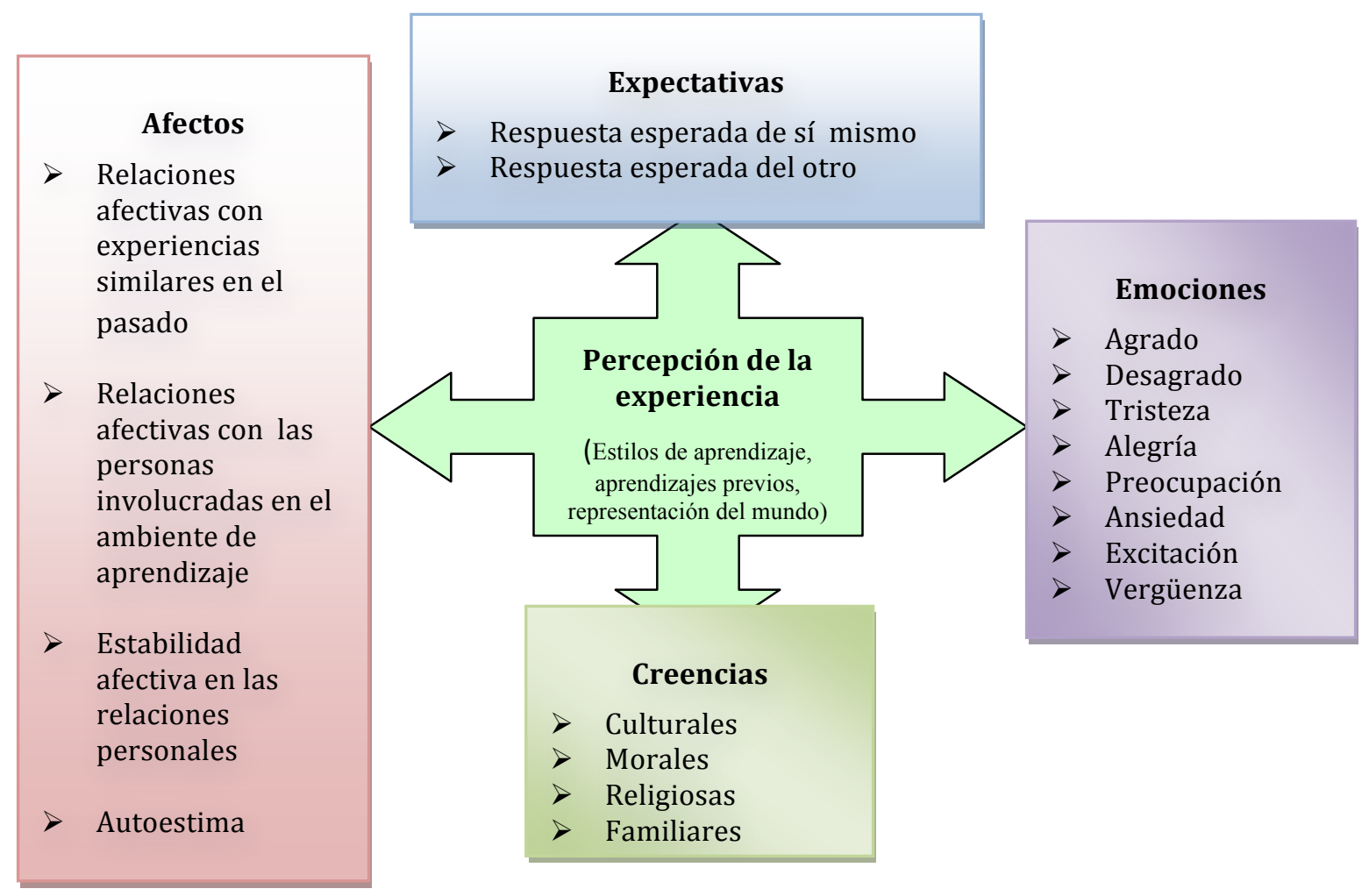

Es fácil estar de acuerdo con Paul Pintrich en cuanto a que "la motivación es evocada para explicar por qué las personas consiguen hacer algo, se mantienen haciéndolo y se ayudan a sí mismas para llevarlo hasta el final” (2003, p.104), sobre lo que existen varias teorías; pero aquí interesa tratar lo comentado por el autor acerca del papel de los componentes motivacionales para el aprendizaje.

Pintrich destaca tres modelos importantes: el primero tiene que ver con las habilidades o destrezas que uno mismo posee para desempeñar la tarea (expectativas de los componentes); el segundo se refiere a las creencias acerca de la importancia, interés y utilidad de la tarea (valor de los componentes); el tercero hace mención a los sentimientos o reacciones emocionales que la tarea provoca en uno mismo. 
En relación con el primer modelo, el estudiante se pregunta si será capaz de hacer esa tarea. En este caso, distintos autores hablan de la importancia de las creencias de control o de autodeterminación, o sea, de que el estudiante cuente con la motivación intrínseca (no controlada por otros) que surge al sentirse capaz de cumplir con la tarea. Condición que lo ayuda a asumir sus propias responsabilidades, a ser persistente cuando no lo logra y a mejorar su desempeño cognitivo, su rendimiento y su autoestima.

Por el contrario, cuando el alumno no encuentra una posible contingencia entre su comportamiento y las demandas de la tarea, debido a que depende del control de los otros (locus de control externo) puede mostrarse ansioso, pasivo, sin ganas de hacer esfuerzos. Otra de sus características es tender a atribuir su falta de logro a causas inestables, como el nivel de dificultad de la tarea o la mala suerte, que de seguro no sólo lo llevarán a dar un bajo rendimiento, sino a culpar a los otros de su fracaso, a asumir que nada está bajo su control y a que es incapaz por sí mismo de actuar positivamente sobre el ambiente. En estos casos, los educadores pueden ayudar mucho a sus estudiantes sobre sus percepciones de control, dándoles guías claras, consistentes y, por supuesto, ajustadas a la capacidad ya evaluada de los alumnos, así como proveerles de una constante realimentación positiva.

La teoría también trata de las creencias de auto-eficacia, referidas a los juicios que tiene el estudiante sobre su habilidad para cumplir con las tareas pero en ciertos campos específicos. Él o ella se sabe más eficiente en algunas áreas que en otras y esto ayuda mucho cuando se requiere hacer trabajo de equipo, en el que los miembros se regulan y se enseñan unos a otros, como en el caso de las actividades colaborativas y otras que anotaremos más adelante. Además, Pintrich asegura que la auto-eficacia es un predictor 
positivo de auto-regulación en el estudiante, así como de su compromiso por seguir conociendo acerca de algo, también reporta que estudiantes con un adecuado manejo de la auto-eficacia utilizan estrategias de mayor elaboración, organización y profundización cognitiva, definitivamente muy necesarias de remarcar en los estudiantes con DATH.

Con respecto al modelo del valor de los componentes, el mismo autor menciona que posiblemente el estudiante se pregunte ¿por qué yo estoy haciendo esta tarea?; lo que le permite señalar, como investigador, que su modelo consta de dos elementos básicos que son la orientación hacia la meta y, propiamente, el valor de la tarea.

La orientación hacia la meta puede poner la mirada directamente en el punto de llegada, lo que parece ser una actividad más propia del estudiante, o bien, él o ella puede plantearse una especie de metas por tareas o pasos específicos, que comúnmente son mediados por otros, como es el caso de los docentes. En este campo hay una discusión importante sobre qué será lo mejor, pero en realidad parece que una forma funciona más con unos estudiantes que con otros. Lo importante aquí es poder lograr que el educando domine la tarea, lo que parece estar vinculado con adaptación cognitiva, uso de estrategias de autoregulación y aprendizaje más profundo.

En el caso del valor de la tarea, éste puede ser aprendido, con convencimiento, del valor que otros le dan a la misma (motivación extrínseca) o gracias a la utilidad a corto o largo plazo que los estudiantes identifican en ese aprendizaje, lo que lleva a una motivación intrínseca. Con respecto a la motivación extrínseca se identifican cuatro niveles: i) cuando el estudiante espera recibir una recompensa o evitar un castigo por parte del otro; ii) cuando cumple con la tarea para no sentirse culpable, debido a que si no tiene un buen rendimiento 
hará sentirse mal a otros; iii) cuando el estudiante quiere hacer bien la tarea más por una condición utilitarista con respecto a otros que por propio interés y iv) cuando el estudiante integra a su propio esquema los esquemas de otros, ya que estos enriquecen el valor que le da a la tarea, pero esta integración es una decisión totalmente autónoma.

De esta manera, se reconoce que "los más altos valores del nivel de la tarea (importancia, interés y utilidad) están asociados con adaptación cognitiva, altos niveles de uso de estrategias de autorregulación y más altos niveles de rendimiento” (Pintrich, 2003, p.114).

Cuando se trata de los componentes afectivos, como señalábamos al inicio de este apartado, éstos pueden facilitar o disminuir las posibilidades del conocimiento y el aprendizaje. Las emociones podrían afectar la recuperación o el almacenamiento de información en la memoria, sobre todo de la memoria de trabajo, así como los procesos de codificación y asociación. También, pueden afectar negativamente al requerimiento de procesos de información más analíticos y detallados, lo que se asocia con una mayor probabilidad de cometer errores y alcanzar elaboraciones cognitivas poco profundas. De hecho, algunos sujetos con DATH u otro tipo de problema de aprendizaje, acuden a evitar el fracaso, poniendo excusas ante las pruebas, refugiándose siempre en tareas sencillas o atribuyendo el fracaso a la falta de tiempo de estudio.

Esas reacciones requieren de estrategias de auto-regulación que si no se dominan derivan en menos aprendizaje y disminución en los niveles de desempeño. Pintrich (2003) sugiere que los maestros necesitan estar conscientes del papel de la ansiedad en la disminución del rendimiento, y tratar de reducir los efectos potenciales de debilitamiento en sus propias acciones de mediación. Por otra parte, la mayoría de los autores están de acuerdo en sugerir 
que los individuos siempre necesitan estar motivados para establecer, mantener y promover una autoimagen positiva.

\section{Procesos de auto-regulación}

Ya ha sido comentado que uno de los problemas que más afectan a los procesos de aprendizaje es la falta de auto-regulación de los impulsos, sobre todo de aquellos que llevan a moverse sin control y a cometer conductas inapropiadas, lo que redunda en la falta de atención y así de comprensión y retención de los aprendizajes, sin contar el permanente rechazo de los otros. Por eso, tratar el tema de auto-regulación para ganar en procesos de atención y concentración cuando se trata de estudiantes con DATH es indispensable.

La auto-regulación ha sido estudiada desde varias perspectivas teóricas y una de ellas es la conductista. En ese caso, se selecciona la conducta que se desea trabajar, se hace un programa de auto-monitoreo, de auto-instrucción y de recompensa. Funciona muy bien con niños mayores o personas en general que tienen problemas para mantenerse estudiando un tiempo prudencial. Por ejemplo, se plantea la conducta de mantenerse estudiando al inicio unos 40 minutos y se eliminan todos los posibles distractores del lugar de estudio, cuando lo ha logrado puede acudir a la recompensa y poco a poco es posible ir aumentando el tiempo de estudio y administrar la recompensa de distintas maneras.

Para el auto-monitoreo, debemos estar seguros de que el estudiante, primero, está consciente de que tiene un problema con su tiempo de estudio y, segundo, que es capaz de chequear su propio comportamiento. También puede utilizarse la frecuencia con la que ocurre una conducta. En ese caso se chequea el número de veces, ya sea porque existe una intención de aumentarla -como en el caso de dirigirse voluntariamente al cuarto de estudio- 
o de disminuirla -como el número de veces que se pone de pie para interrumpir a sus compañeros o simplemente a sí mismo.

La auto-instrucción requiere de claves llamativas en el ambiente que ayuden al estudiante con DATH a recordar las cosas que debe hacer. Pueden ser anotaciones hechas en lugares ineludibles o desarrollar el hábito de escribir y revisar una agenda. Schunk y Zimmerman (2003) nos recuerdan que las auto-instrucciones son muy útiles en ejercicios como la comprensión lectora y la escritura precisa, en tanto enseñan al estudiante a detenerse cada cierto tiempo durante la actividad para hacerse preguntas clave, que incluso puede señalar en el texto de forma llamativa. Las frases pueden ir secuenciadas y decir cosas como: ;debo volver a leer la pregunta!, ¿sobre quién habla el texto?, o, ¡leo en voz alta cada palabra que escribi!!

Cuando se trabajan procesos de auto-regulación, se aconseja que, al menos al inicio, la recompensa sea administrada por otro; luego, sobre todo en el caso de jóvenes mayores o adultos, es posible pasar pronto a procesos de auto-recompensa.

Las estrategias que utiliza la teoría del procesamiento de la información en estos casos requieren que el estudiante tenga algún dominio de procesos metacognitivos, ya que tiene que estar consciente de cuáles son las demandas de la tarea, cuáles son sus capacidades para resolverla y cuáles son los puntos de la tarea que podrían demandarle algunas estrategias especiales para poder completarla. Por lo general, se componen de dos pasos básicos: a) comparaciones de la actividad contra una solución estándar y b) tomar tiempo para resolver las discrepancias con respecto al estándar. Pero la clave principal está en ir encadenando fases de la actividad, que se sostiene en ese momento en la memoria de trabajo, con la 
información almacenada en la memoria a largo plazo; por eso, detenerse para comparar y resolver es muy necesario.

Bajo esta teoría, una estrategia igualmente importante para aprender a partir de un texto es el ensayo (rehearsal), que incluye repetir información, subrayar y resumir (Schunk y Zimmerman, 2003). No obstante, es útil advertir que para subrayar el estudiante debe primero dar evidencia de que identifica las ideas más importantes y las secundarias (orden jerárquico). De otra manera, se corre el riesgo de que aparezca un texto casi totalmente subrayado, lo que implica que no hay criterio de orden para almacenar la información. Lo mismo puede ocurrir cuando se pide resumir. Al inicio puede ayudar el hecho de poner un espacio reducido para obligar al estudiante a colocar sólo las ideas principales, pero para llegar a ese punto debe haberlas dicho oralmente con sus propias palabras y luego escribirlas. Puede que sea necesario aplicar el procedimiento paso a paso con cada una de las ideas.

Otra estrategia útil dentro del procesamiento de la información es la elaboración, que consiste en usar imágenes, acrósticos o llaves con letras de inicio como ayudas mnemotécnicas para recordar información. También es útil el detenerse a menudo y hacerse preguntas de lo que se está aprendiendo, lo mismo que tomar notas sobre cosas importantes de recordar cuando que el asunto se trae a la conciencia. Otra clave de apoyo es utilizar rotulaciones y bosquejos en los márgenes de los textos y el mapeo. Pero, hay que estar claros en que un mapa conceptual, por ejemplo, requiere de formas complejas de razonamiento a partir de relaciones proposicionales que no todos los estudiantes con DATH llegan a dominar. 
El monitoreo de comprensión debe realizarse, por ejemplo, haciéndose constantes preguntas a sí mismo, releyendo, completando listas de cotejo y parafraseando la información. Además, es importante no olvidar el planteamiento de metas y convencer a los estudiantes de que el logro siempre los guiará hacia metas de mayor alcance. De la misma manera, es posible tener dentro de la población destinataria de las mediaciones de aprendizaje a aquellos estudiantes que reaccionan positivamente a la realimentación mediante la comparación social. Por lo que puede ser útil aplicar consignas o frases de animación social, como por ejemplo: todos los compañeros que llegaron hasta aquí pudieron alcanzar la meta siguiente, entonces usted también lo va a lograr.

\section{La memoria y los procesos de información}

Desde el enfoque constructivista, Mayer (2003) da una explicación particular:

El conocimiento es una representación mental que existe en la mente humana. Que se distingue de la información, en que esta es una entidad objetiva que puede ser trasladada de una mente a otra, mientras que el conocimiento es una construcción personal que no puede ser directamente movilizada de una mente a otra. (p.49)

En este caso, el conocimiento está mediado por el entorno cultural del aprendiz y por la forma individual en que este procesa su nuevo aprendizaje, en función también de los aprendizajes previos adquiridos, donde también influye su estado motivacional y emocional, que es individual para cada sujeto. Por esa razón, asegura el autor, a diferencia de la información que pasa tal y como se emite de un sujeto a otro, el conocimiento es siempre reinterpretado. 
Mientras tanto, a la teoría del procesamiento de la información se le reconoce que uno de sus mayores aportes ha sido el del análisis de tareas. O sea, permitir desglosar en pasos el proceso que se sigue para desarrollar la tarea. Lo que se considera muy útil en el caso de estudiantes con DATH, ya que permite identificar los pasos que un estudiante debe aprender, así como aquellos que le pueden estar presentando dificultades.

De acuerdo con esta teoría: "El aprendizaje es la construcción del conocimiento; la memoria es el almacenamiento del conocimiento y el pensamiento es la manipulación lógica de ese conocimiento" (Mayer, 2003, p.50). Por otra parte, el conocimiento se constituye de representaciones mentales de distinto tipo, que pueden ser clasificadas por la forma en cómo éstas ingresan mediante nuestro sistema neurosensorial o por la forma en que esas representaciones pasan por la memoria de trabajo, establecen relaciones entre sí y, finalmente, se integran para instalarse en la memoria a largo plazo como un aprendizaje significativo. Por esa razón, también es relevante considerar que el óptimo estado de los canales sensoriales, es condición esencial para el procesamiento mental requerido en un aprendizaje exitoso. 


\section{FIGURA 3}

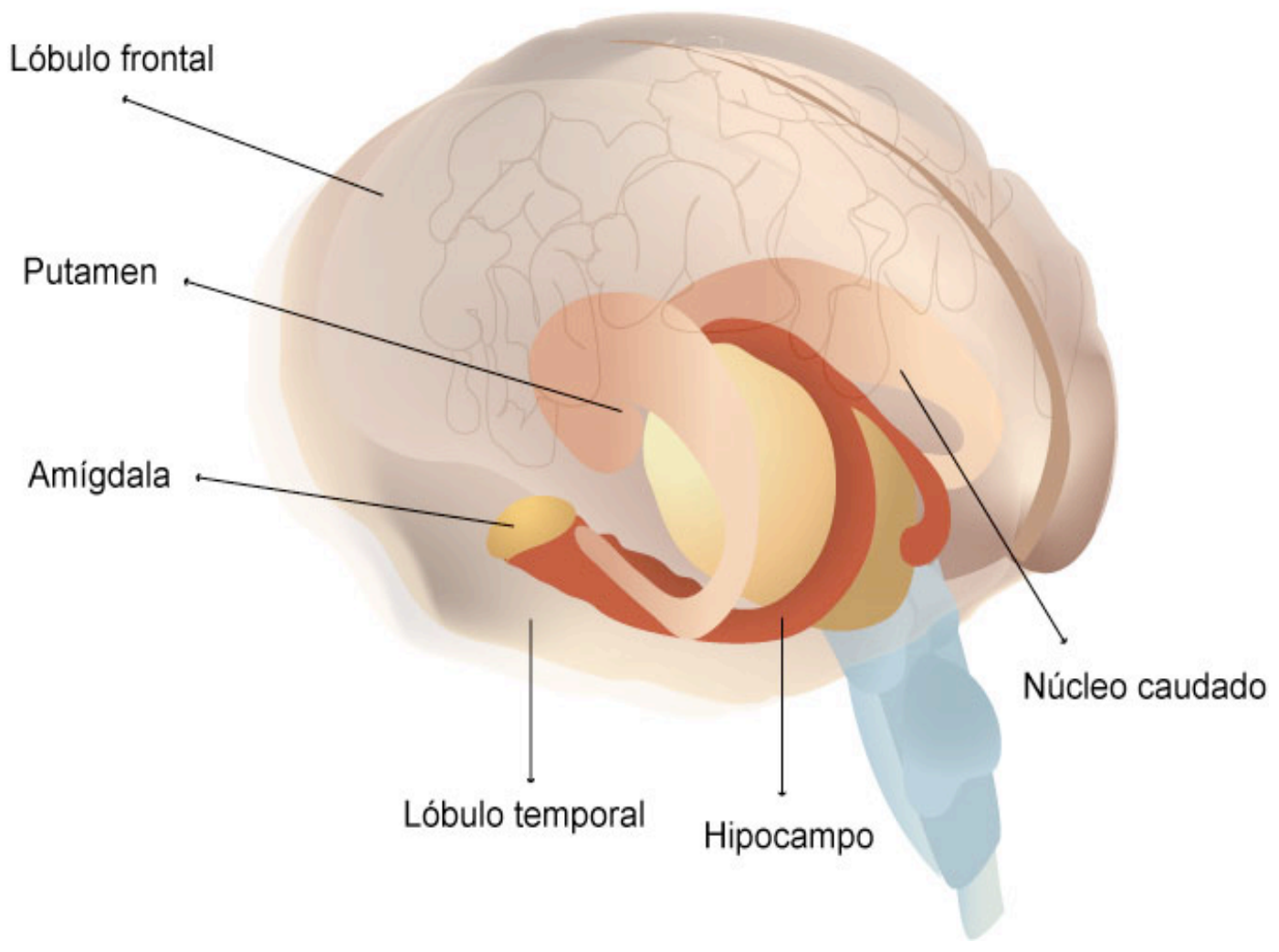

Tomada y adaptada de Carter (2002).

La memoria es un sistema sumamente complejo, en el que intervienen una serie de áreas diferentes del cerebro, tanto del sistema límbico como de la neocorteza. Por ejemplo, muchos instintos están grabados genéticamente en el núcleo caudado, el hipocampo interviene en la recuperación de casi todo tipo de recuerdos, la amígdala almacena memorias traumáticas. El putamen se encarga de guardar memorias de procedimiento, el lóbulo temporal memorias de largo plazo y el lóbulo frontal memorias de corto plazo como es el caso de las memorias de trabajo. Aquí se muestra, además, la cercanía y la mutua influencia que existe entre el sistema emocional (límbico) y el sistema de memoria en el cerebro humano.

Resulta esencial comprender que atender a los estudiantes con DATH debe hacerse desde un enfoque inclusivo, lo que implica resolver sus dificultades mientras cuentan con 
oportunidades equitativas, eficientes y compartidas con el resto de sus compañeros, para desarrollar los aprendizajes que exige el sistema educativo en que se forma. En ese caso, las metodologías que mejor favorecen dicho propósito son aquellas que buscan alcanzar el desarrollo de habilidades de auto-motivación, auto-regulación y memoria en un ambiente colaborativo. Como sugerencia, es posible mencionar actividades relacionadas con: aprendizaje por proyectos, aprendizaje multimodal (combinando los distintos estilos de aprendizaje), aprendizaje multinivel, resolución interactiva de problemas y análisis de casos, entre otras.

Además, debemos considerar que el uso de las nuevas tecnologías de la información y la comunicación puede favorecer el desarrollo de este tipo de actividades y que existe software libre y gratuito sobre el tema (Sánchez, 2006) ${ }^{2}$. Al respecto, es necesario poner énfasis en que estas metodologías se prestan para ser muy bien alineadas en los materiales mediacionales que se emplean en la educación a distancia, pero que obligan a crear formas de monitoreo que, por supuesto, no pueden ser las mismas que se utilizan en los medios presenciales.

Sobre la descripción y fines específicos de estas actividades valdría la pena extenderse, pero no lo permiten los límites de este trabajo, por lo que es necesario informar acerca de que actualmente hay gran variedad y cantidad de fuentes que nos orientan y dan ideas sumamente creativas con el propósito de llevarlas a cabo, para lo que se sugiere revisar Barkley et al. (2007); Barnet, et al. (2003); Ferreiro (2007); Howard-Jones (2006), Prendes (2003); Puigdellìvol (2005) y Pujolàs (2003). 


\section{CONCLUSIONES}

La neuroimaginería de los últimos años ha permitido a los investigadores observar las distintas reacciones y funciones cerebrales mientras ocurren (on time). Las implicaciones de esta posibilidad son infinitas en cuanto a nuevos aportes al conocimiento en diversos campos. En nuestro caso, nos interesa el campo de la educación y la dinámica de la fisiología cerebral mientras se aprende o de sus alteraciones mientras se debería aprender y no se logra. Más específicamente, en esta oportunidad, para el desarrollo de este trabajo seleccionamos las dificultades de aprendizaje que exhiben las personas con DATH y cómo se representan éstas neurofisiológicamente.

Al respecto, recordamos que las personas con este déficit presentan problemas de impulsividad, de control de sus movimientos, distracción y oposición a las normas, entre otras características. Este repertorio deriva en dos grupos de problemas que se confabulan en contra de la persona que intenta aprender, uno tiene que ver con el rechazo social que esos comportamientos provocan y el otro se refiere a sus consecuencias negativas sobre el rendimiento escolar.

Los nuevos hallazgos permitidos por la neuroimaginería dejan ver que lo que se juzgaba como un comportamiento oposicional volitivo o malintencionado responde, más bien, a un déficit en el desarrollo de la corteza prefrontal y de los ganglios basales, ambos relacionados con la auto-regulación; y que, a las dificultades para aprender les subyace la falta de integración de distintas partes del lóbulo temporal que son dependientes entre sí. Estas observaciones señalan el camino para propuestas educativas sistemáticamente 
diferentes y más atinadas que las que se han venido aplicando hasta ahora en la atención educativa dirigida a personas con DATH.

Por otra parte, la neuroimaginería también ha permitido confirmar que el castigo y la exclusión, como respuesta a la conducta desafiante y desatenta de los estudiantes con DATH, deprime acciones fisiológicas asociadas con el aprendizaje exitoso, y que, por el contrario, la motivación extrínseca e intrínseca dispone el desarrollo de estructuras cerebrales propias, alternas o compensatorias en las que toman lugar los distintos procesos para aprender; lo que nos ha llevado a explicar formas de animar la auto-regulación y la motivación, asociadas a diversas tareas cognitivas.

Por último y de manera relevante, es necesario destacar que las actividades de aprendizaje colaborativo resultan estratégicas en este caso, en tanto propician la regulación social y la auto-regulación en las relaciones con otros, animan la observación, la previsión, la planificación, la resolución de problemas y la toma de decisiones, además de favorecer el logro de los objetivos mediante tareas compartidas y, con esto, la motivación para continuar aprendiendo. Lo que indica, que las estrategias de aprendizaje colaborativo reúnen las condiciones necesarias para educar apropiada e inclusivamente a los estudiantes con DATH.

\section{NOTAS}

1 Los grandes avances de la tecnología de la neuroimaginería (también referida como neuroimagería) han impulsado conocimientos impredecibles en todas las áreas del desarrollo humano. Entre las técnicas de neuroimaginería, cada vez más sofisticadas, se encuentran: la electroencefalografía (EEG), la Magnetoencefalografía (MEG), la Tomografía Axial Computarizada (TAC), la Resonancia Magnética (RM), la Resonancia Magnética Funcional (RMf), la Tomografía por Emisión de Positrones (TEP) y la Estimulación Magnética Transcraneana (EMT). La nueva tecnología de neuroimagen permite estudiar las reacciones o no del sistema nervioso en vivo ante los diferentes estímulos, entre las que se incluye el comportamiento neurofisiológico ante las distintas situaciones de aprendizaje (Blakemore y Frith, 2007 y Carlson, 2007). 
2 Es posible encontrar referencias actualizadas y útiles en el sitio web de Rafael Sánchez Montoya, investigador de la Universidad de Cádiz: www.ordenadorydiscapacidad.net, así como en estos otros sitios: www.nalejandria.com/akademeia/, www.copgasteiz.com, www.educared.net.

\section{REFERENCIAS}

American Psychiatric Association (1994). DSM-IV-TR. Estados Unidos de América: [s.n.].

Barkley, E.; Cross, K.; Howell, C. (2007). Técnicas de aprendizaje colaborativo. Madrid: Morata.

Barnett, L.; Echeíta, G.; Escofet, N.; Fernández, C.; Guix, M.D.; Jiménez, J.R.; López, G.; Lloret, F.; Maté, M.; Martín, M.; Mir, C.; Ojea, M.; Pujolàs, P.; Redó, M.; Rué, J.; Serra, P. y Solsona, N. (2003). Motivación, tratamiento de la diversidad y rendimiento académico. El aprendizaje cooperativo. Barcelona: GRAÓ.

Blakemore, S. J.; Frith, U. (2007). Cómo aprende el cerebro. Las claves para la educación. Barcelona: Ariel.

Carlson, N. (2007). Fisiología de la conducta. Massachusetts: Pearson.

Carter, R. (2002). El Nuevo mapa del cerebro. Barcelona: Integral.

Ferreiro, R. (2007). Nuevas alternativas de aprendizaje y enseñanza. Madrid: Trillas.

Howard-Jones, P. (2006). Neuroscience and education: issues and opportunities. A commentary by The Teaching and Learning Research Programme. En TLRP-ESRC Seminar Series, Collaborative Frameworks in Neuroscience and Education. UK: Institute of Cognitive Neuroscience, University College London.

Mayer, R. (2003). Memory and Information Process. Handbook of Psychology. Vol. 7. Educational Psychology: Chapter 3. Canadá: John Wiley and Sons \& Inc.

Meléndez, L. (2004). Estrategias de desarrollo del pensamiento para la superación de limitaciones intelectuales. II Congreso Internacional de Discapacidad Intelectual, Medellín, Colombia.

Organización Mundial de la Salud (2001). Clasificación Internacional del Funcionamiento, de la Discapacidad y de la Salud (CIF). España: IMSERSO. 
Perea, M. V. (2008). Rehabilitación cognitiva. Conferencia Inaugural del Doctorado en Neuropsicología, Universidad de Salamanca-Universidad Iberoamericana. Costa Rica: UNIBE.

Pintrich, P. (2003). Motivation and classroom learning. Handbook of Psychology, 7. Educational Psychology: Chapter 6. Canada: John Wiley and Sons \& Inc.

Prendes, M. P. (2003). Aprendemos... ¿Cooperando o colaborando? Las claves del método. En F. Martínez, Redes de Comunicación en la Enseñanza. Las nuevas Perspectivas del Trabajo Colaborativo. Barcelona: Paidós Ibérica, S.A.

Puigdellìvol, I. (2005). La educación especial en la escuela integrada. Una perspectiva desde la diversidad. Madrid: GRAÒ.

Pujolàs, P. (2007). Aulas inclusivas y aprendizaje cooperativo. Novedades Educativas, 19 (202), 21 29.

Sánchez, R. (2006). Las tecnologías en la escuela inclusiva. Murcia: Gobierno de Murcia y Real Patronato sobre Discapacidad.

Schunk, D.; Zimmerman, B. (2003). Self-regulation and learning. Handbook of Psychology, 7. Educational Psychology: Chapter 3. Canada: John Wiley and Sons \& Inc. 